\title{
ANTIMICROBIAL AND ANTIOXIDANT ACTIVITIES OF TWO ENDEMIC PLANTS FROM AKSARAY IN TURKEY
}

\section{Meltem Asan Ozusaglam ${ }^{1 *}$, Derya Onal Darilmaz ${ }^{1}$, Mahmut Erzengin ${ }^{2}$, Mehtap Teksen ${ }^{3}$, Seher Karaman Erkul ${ }^{3}$}

\author{
${ }^{1}$ Department of Biotechnology and Molecular Biology, Faculty of Art and Science, Aksaray \\ University, Aksaray, Turkey, ${ }^{2}$ Department of Chemistry, Faculty of Art and Science, Aksaray \\ University, Aksaray, Turkey, ${ }^{3}$ Department of Biology, Faculty of Art and Science, Aksaray \\ University, Aksaray, Turkey \\ *E-mail: meltemozusaglam@gmail.com
}

\begin{abstract}
This study was designed to examine the in vitro antimicrobial and antioxidant activities of the methanol, ethanol, water, n-hexane and dicholoromethane extracts of two Allium species (Allium scabriflorum and Allium tchihatschewii) which are endemic for the flora of Turkey. The antimicrobial efficiency of the plant was evaluated according to disc diffusion and microdilution broth methods. The antimicrobial test results showed that the extracts of A. scabriflorum and A. tchihatschewii showed varying degrees of antimicrobial activity on the tested microorganisms. The extracts were screened for their possible antioxidant activities by three complementary tests; DPPH free radical-scavenging, scavenging of hydrogen peroxide and metal chelating activity assays. All the extracts of A. scabriflorum and A. tchihatschewii exhibited lower DPPH free radical scavenging activity but higher metal chelating activity when compared to standards. The values of scavenging of hydrogen peroxide of the extracts were higher than the standards that of $\alpha$-tocopherol, BHA, BHT and trolox, but close to that of ascorbic acid. In addition to the antioxidant activity of these plants, the total phenolic compounds and flavonoids were also measured in the extracts. The results presented here may suggest that the extracts of A. scabriflorum and A. tchihatschewii possess antimicrobial and antioxidant properties, and therefore, they can be used as a natural preservative ingredient in food and/or pharmaceutical industry.
\end{abstract}

Key words: Allium scabriflorum, Allium tchihatschewii, antimicrobial activity, antioxidant activity, phenolic

\section{Introduction}

The spoilage and poisoning of foods by microorganisms are problems that have not yet been brought under adequate control despite the range of robust preservation techniques available. Raw and/or processed foods are open to contamination during the production, sale and distribution of them (Deak and Beuchat, 1996). Thus, at present, it is a necessity to use the chemical preservatives to prevent the growth of food spoiling microbes in the food industry (Sağdıç and Özcan, 2003). Consumers are increasingly avoiding foods prepared with preservatives of chemical origin, and natural alternatives are therefore needed to achieve sufficiently long shelf life of foods and a high degree of safety with respect to foodborne pathogenic microorganisms (Rauha et al., 2000).

Synthetic antioxidants and antimicrobials in use have been shown to have harmful side effects (Gao et al., 1999; Williams et al., 1999; Osawa and Namiki, 1981), therefore, there is a need for more effective, less toxic and cost effective antioxidants and antimicrobials from natural sources. Recently, there are a lot of reports on the antioxidant and antimicrobial activities of plant-based extract, which has a potential application both in the food and in medicinal industries (Zeng et al., 2011).

Since ancient times, several species of the genus Allium have been used as foods, spices or herbal remedies. The genus Allium L. is a member of the family Liliaceae and probably the largest genus of the petaloid monocotyledons, comprising some 780 species (Friesen et al., 2006). Allium is naturally distributed in the northern hemisphere and South Africa, mainly in seasonally dry regions. The genus Allium comprises 168 species (188 taxa) in the Flora of Turkey (Davis et al., 1988; Kollmann, 1984; Özhatay and Tzanoudakis, 2000). 73 of them are endemic (Özhatay and Kültür, 2006; Özhatay et al., 2009; Özhatay et al., 2011). Generally, all plant parts of Allium may be consumed by humans (except perhaps the seeds), and many wild species are exploited by the local inhabitants (Fritsch and Friesen, 2002). Anectodal evidence supports the important roles of the members of this genus in the prevention and treatment of pathogenic infections, tumors and cardiovascular diseases. Antioxidative activity of some Allium species has been reported elsewhere (Cao et al., 1996; Gazzani et al., 1998; Yin and Cheng, 1998).

As far as our literature survey could ascertain, A. scabriflorum Boiss. and A. tchihatschewii Boiss., the endemic plants in Aksaray-Turkey, have not been chemically or biologically investigated. Therefore, the aims of the present study were to estimate phenolic and flavonoid contents and to evaluate the antibacterial and antioxidant activities of these plants collected from Aksaray. 


\section{Materials and Methods \\ Plant material and extraction}

Flowering samples of A. scabriflorum (voucher No. Teksen 2571 \& Karaman) and A. tchihatschewii (voucher No. Teksen 2614 \& Karaman) were collected during June-July in 2010 from Genç Osman Village (an altitude of ca. $1050 \mathrm{~m}$ ) and Aksaray University Campus Area, Aksaray, Turkey (altitude of ca. 950m), respectively. They were dried at room temperature. The plants were identified and deposited at the herbarium of Biology Department, Faculty of Science and Art, Aksaray University, Turkey.

The air-dried and powdered plant materials (15 g of each) were extracted seperately with ethanol, methanol, water, n-hexane and dicholoromethane (DCM) by Soxhlet extraction for 24 hours. The extracts were filtered and evaporated by using a rotary evaporator (Heidolph, Laborota 4000, Schwabach, Germany) and stored in the dark at $4^{\circ} \mathrm{C}$ until used within a maximum period of one week.

\section{Determination of antimicrobial activity Test organisms}

Different extracts of various plant parts of A. scabriflorum and A. tchihatschewii were individually tested against a set of 13 microorganisms. The following microorganisms were used in the screening of antimicrobial activity: Seven gram negative bacteria, namely Escherichia coli ATCC 11229, Escherichia coli ATCC 35218, Escherichia coli O157:H7, Salmonella enteritidis ATCC 13076, Pseudomonas aeruginosa ATCC 27853, Shigella sonnei Mu:57, Yersinia enterocolitica NCTC 11175, four gram positive bacteria namely, Listeria monocytogenes ATCC 7644, Staphylococcus aureus ATCC 25923, Bacillus cereus RSKK 863, Micrococcus luteus NRRL B-4375 and two yeasts, Saccharomyces cerevisiae and Candida albicans ATCC 10231. Bacterial strains were cultured overnight at $37^{\circ} \mathrm{C}$ in Nutrient Agar (NA) and Tryptic Soy Agar (TSA) and yeasts were cultured for $48 \mathrm{~h}$ at $30^{\circ} \mathrm{C}$ in YPD Agar medium.

\section{Antimicrobial assay}

The disc diffusion assay was used to determine the antimicrobial potential of the investigated extracts (Murray et al., 1995). The culture suspensions were adjusted by comparing with $0.5 \mathrm{McF}$ arland. One hundred microlitres of suspension of the test microorganisms were spread on solid media plates. Sterile filter paper discs of $6 \mathrm{~mm}$ diameter were impregnated with $10 \mu \mathrm{L}$ of the extract solution. The plates were kept for 2 hours in refrigerator to enable prediffusion of the extracts into the agar. Then the inoculated plates were incubated at $37{ }^{\circ} \mathrm{C}$ for $24 \mathrm{~h}$ for bacterial strains and $30{ }^{\circ} \mathrm{C}$ for $48 \mathrm{~h}$ for yeasts. Ampicillin (Amp, $10 \mu \mathrm{g} / \mathrm{disc})$, Gentamicin (CN, $10 \mu \mathrm{g} / \mathrm{disc}$ ) and Amikacin (AK, $30 \mu \mathrm{g} / \mathrm{disc})$ were used as positive controls. Negative controls were performed with paper discs loaded with $10 \mu \mathrm{L}$ of solvents (ethanol, methanol, water and dimethyl sulfoxide (DMSO)). At the end of the incubation period the antimicobial activity was evaluated by measuring the inhibition zones (the diameter of inhibition zone plus the diameter of the disc).

\section{Broth microdilusion assay for minimum inhibitory concentrations (MICs)}

The antimicrobial activity of the extracts was also evaluated through the determination of the MIC by the microdilution method in culture broth with some modifications (Koneman et al., 1997). The minimal inhibitory concentration (MIC) values were studied for the microorganisms, being sensitive to the extracts in the disc diffusion assay. Tubes were prepared by dispensing $95 \mu \mathrm{L}$ of growth medium, $100 \mu \mathrm{L}$ of the extract and $5 \mu \mathrm{L}$ of the inoculum. The serial dilutions of the extracts were done in a concentration range from 1.41 to $180 \mu \mathrm{g} / \mu \mathrm{L}$ in the tubes. The inoculum of microorganisms was prepared using $12 \mathrm{~h}$ cultures and suspensions were adjusted to $0.5 \mathrm{McFarland}$ standard turbidity. The final volume in each tube was $200 \mu \mathrm{L}$. A positive control (containing $5 \mu \mathrm{L}$ inoculum and $195 \mu \mathrm{L}$ growth medium) and a negative control (containing $100 \mu \mathrm{L}$ of extract, $100 \mu \mathrm{L}$ growth medium without inoculum) were included on each microtube. The contents of the tubes were mixed by pipetting, and they were incubated at appropriate temperatures for $24 \mathrm{~h}$. Then, $5 \mu \mathrm{L}$ samples from clear tubes were plated on solid growth medium to confirm microbial growth because the plant extracts tested in this study were colored (Şahin et al., 2003). The MIC was defined as the lowest concentration of the compounds needed to inhibit the growth of microorganisms. Each test was repeated at least triplicate.

\section{Antioxidant capacity \\ DPPH radical scavenging activity}

The radical scavenging activity was determined by a spectrophotometric method based on the reduction of ethanol solution of DPPH (Blois, 1958). The extracts were dissolved in ethanol $(1 \mathrm{mg} / \mathrm{mL})$, and various concentrations $(5,25,50$ and $75 \mu \mathrm{g} / \mathrm{mL}$ ) of each extract were used. Similar concentrations of $\alpha$-tocopherol, BHA, BHT and ascorbic acid were used as positive control. The prepared extracts were transferred in tubes and then total volume was adjusted to $1.5 \mathrm{~mL}$ with ethanol. $0.5 \mathrm{~mL}$ of $0.1 \mathrm{mM}$ solution of DPPH radical in ethanol DPPH radical was added to each test tube, and the mixture was shaken vigorously. After $30 \mathrm{~min}$ incubation at room temperature in the dark, the decrease in absorbance was measured at 517 $\mathrm{nm}$. The radical scavenging activity was calculated from the equation [1]:

$\%$ of radical scavenging activity $=\left[\left(\mathrm{Abs}_{\mathrm{control}}-\mathrm{Abs}_{\mathrm{sample}}\right) / \mathrm{Abs}_{\mathrm{control}}\right] \times 100$

wherein $\mathrm{Abs}_{\text {control }}$ is the absorbance of the control reaction, and $\mathrm{Abs}_{\text {sample }}$ is the absorbance in the presence of the test compound. 


\section{Metal chelating activity}

The chelating of ferrous ions by the extracts and standards was estimated according to the method of Dinis et al. (1994) with some modifications. Briefly, $30 \mu \mathrm{g} / \mathrm{mL}$ concentration of the each extract in ethanol was added to a solution of 2 $\mathrm{mM} \mathrm{FeCl}_{2}(25 \mu \mathrm{L})$. Then, total volume was adjusted to $1.9 \mathrm{~mL}$ with ethanol. The reaction was initiated by the addition of 5 $\mathrm{mM}$ ferrozine $(0.1 \mathrm{~mL})$, and the mixture was shaken vigorously and left to stand at room temperature for $10 \mathrm{~min}$. The absorbance of the solution was then measured spectrophotometrically at $562 \mathrm{~nm}$. $\alpha$-tocopherol, BHA, BHT, ascorbic acid, trolox and EDTA were used as positive controls. The percentage of inhibition of ferrozine- $\mathrm{Fe}^{2+}$ complex formation was calculated using the equation [1].

\section{Scavenging of hydrogen peroxide}

The ability of the extracts to scavenge hydrogen peroxide was determined according to the method of Ruch et al. (1989). A solution of hydrogen peroxide (43 mM) was prepared in $0.1 \mathrm{M}$ phosphate buffer (pH 7.4). $30 \mu \mathrm{g} / \mathrm{mL}$ concentration of the each extract in ethanol was added to a solution of $43 \mathrm{mM} \mathrm{H}_{2} \mathrm{O}_{2}(130 \mu \mathrm{L})$. Then, total volume was adjusted to $1.0 \mathrm{~mL}$ with $0.1 \mathrm{M}$ phosphate buffer $(840 \mu \mathrm{L})$. The absorbance of hydrogen peroxide at $230 \mathrm{~nm}$ was determined after 30 min against a blank solution containing phosphate buffer without hydrogen peroxide. For each concentration, a separate blank sample was used for background subtraction. $\alpha$-tocopherol, BHA, BHT, ascorbic acid and trolox were used as positive controls. The percentage of scavenging activity of hydrogen peroxide was calculated using the equation [1].

\section{Determination of the amount of phenolic compounds and flavonoids}

The total phenolic content was estimated by the Folin-Ciocalteu colorimetric method, based on the procedure of Singleton and Rossi (1965) with some modification as described below, using gallic acid as a standard phenolic compound. Briefly, $1 \mathrm{~mL}$ solution of extracts containing $1 \mathrm{mg}$ extract in ethanol was mixed with $22 \mathrm{~mL}$ distilled water. $0.5 \mathrm{~mL}$ of FolinCiocalteu reagent was added, and the content of the flask mixed thoroughly. After 3 min, $1.5 \mathrm{~mL}$ of $2 \% \mathrm{Na}_{2} \mathrm{CO}_{3}$ solution was added to the mixture. After incubation for $2 \mathrm{~h}$ at room temperature, the absorbance against blank was measured at $760 \mathrm{~nm}$ with an UV-Vis Spectrophotometer (HITACHI U-2000). Gallic acid was used as a standard for calibration curve. The total phenolic content was expressed as $\mu \mathrm{g}$ gallic acid equivalents $(\mathrm{GAE}) / \mathrm{mg}$ dry extracts. All samples were analyzed in triplicates.

The measurement of total flavonoid content was based on the method described by Park et al. (1997) with slight modifications. $1 \mathrm{~mL}$ of the extract solution containing $1 \mathrm{mg}$ extract was added to a test tube containing $0.1 \mathrm{~mL}$ of aluminum nitrate $(10 \%), 0.1 \mathrm{~mL}$ of aqueous potassium acetate $(1 \mathrm{M})$, and $4.1 \mathrm{~mL}$ of ethanol. After incubation for $40 \mathrm{~min}$ at room temperature, the absorbance was read spectrophotometrically at $415 \mathrm{~nm}$. A calibration curve of quercetin was prepared, and flavonoid contents were determined from the linear regression equation of the calibration curve. The total flavonoid content was expressed as $\mu \mathrm{g}$ quercetin equivalents $(\mathrm{QE}) / \mathrm{mg}$ of dry extracts. All extracts were analyzed in triplicate.

\section{Results and Discussion Antimicrobial activity}

The antimicrobial activities of A. scabriflorum and A. tchihatschewii extracts were employed against the microorganisms and their activity potentials were qualitatively and quantitatively assessed by the presence or absence of inhibition zones, zone diameters and MIC values. The results showed that the A. scabriflorum and A. tchihatschewii extracts those with except water had great potential as antimicrobial agents against the tested microorganisms. The antimicrobial activities of the plants extracted in different solvents varied greatly because there are many factors that influence the active principle present in the plant (Parekh and Chanda, 2006). Here the polarity of the extracting solvents was different, and it greatly influenced the antimicrobial property (Table 1). According to the results given in Table 1, the aqueous extracts of $A$. scabriflorum and A. tchihatschewii did not exhibit any activity against all the tested microorganisms. Similarly, several researchers (Martin, 1995; Paz et al., 1995; Vlientinck et al., 1995) have generally reported that water extracts of plants do not have much activity against bacteria. Durmaz et al. (2006) reported that water extract of $A$. vineale from Bitlis, Turkey had no antibacterial activity against any of nine tested bacteria. DCM extracts of the two species, however, showed antimicrobial activity against 10 out of 13 microorganisms. DCM extracts had the highest inhibitory activities against B. cereus RSKK 863 $(30.24 \mathrm{~mm})$ and $S$. sonnei Mu:57 $(13.17 \mathrm{~mm})$ for A. scabriflorum and A. tchihatschewii, respectively. The results obtained from the disc diffusion method, indicated that $n$-hexane extract of $A$. tchihatschewii exhibited a stronger antimicrobial activity in comparison with the A. scabriflorum n-hexane extract (Table 1). However, all A. scabriflorum extracts could not inhibit the growth of E. coli O157:H7 and S. enteritidis ATCC 13076. P. aeruginosa ATCC 27853 was the most sensitive organism tested to the both plants extracts. Najjaa et al. (2007) reported antimicrobial potential of a wild endemic Allium species growing in the arid region of Tunisia. They found that $A$. roseum essential oil had a high effect against gram negative $P$. aeruginosa, which is a similar result with our study. Especially greater and remarkable antimicrobial activities of dicholoromethane extract were recorded (Najjaa et al., 2011). The results obtained from the controls indicated that the solvents had no effect on the microorganisms.

Some of these plants extracts were more effective than traditional antibiotics (Ampicillin, Amikacin and Gentamicin) to combat the pathogenic microorganisms and some reference strains studied (Table 1). Some A. scabriflorum DCM extracts, for example, have shown better antibacterial activity against 4 out of 11 bacteria when compared with standard Gentamicin and Amikacin (Table 1). An A. scabriflorum ethanol extract exhibited a stronger antibacterial activity in 
http://dx.doi.org/10.4314/ajtcam.v10i3.11

Table 1: Antimicrobial activity of Allium scabriflorum and Allium tchihatschewii extracts

\begin{tabular}{|c|c|c|c|c|c|c|c|c|c|c|c|c|c|c|c|c|c|c|c|c|c|c|c|}
\hline \multirow{3}{*}{ Org. } & \multicolumn{10}{|c|}{ Diameter $^{\mathrm{a}}$ of zone of inhibition $(\mathrm{mm})$} & \multicolumn{10}{|c|}{$\mathrm{MIC}(\mu \mathrm{g} / \mu \mathrm{L})$} & \multirow{2}{*}{\multicolumn{3}{|c|}{$\begin{array}{l}\begin{array}{l}\text { Diameter }^{\text {a }} \text { of zone of } \\
\text { inhibition }(\mathrm{mm})\end{array} \\
\text { Antibiotics } \\
\end{array}$}} \\
\hline & Allium & cabriflor & & & & Allium t & chihatsch & ewii & & & Allium & scabrifl & orum & & & Allium & tchihats & chew & & & & & \\
\hline & M-E & E-E & $\begin{array}{l}\mathbf{W}- \\
\mathbf{E}\end{array}$ & H-E & $\begin{array}{l}\text { DCM- } \\
\text { E }\end{array}$ & M-E & E-E & $\begin{array}{l}\mathbf{W}- \\
\mathbf{E}\end{array}$ & H-E & $\begin{array}{l}\text { DCM- } \\
\text { E }\end{array}$ & M-E & E-E & $\begin{array}{l}\mathbf{W}- \\
\mathbf{E}\end{array}$ & H-E & $\begin{array}{l}\text { DCM- } \\
\text { E }\end{array}$ & M-E & $\mathbf{E}-\mathbf{E}$ & $\begin{array}{l}\mathbf{W}- \\
\mathbf{E}\end{array}$ & H-E & $\begin{array}{l}\text { DCM- } \\
\text { E }\end{array}$ & Amp & AK & $\mathbf{C N}$ \\
\hline 1 & - & - & - & $\begin{array}{l}10.03 \pm \\
0.52\end{array}$ & $\begin{array}{l}13.10 \pm \\
0.03\end{array}$ & - & - & - & $\begin{array}{l}10.36 \pm \\
0.31\end{array}$ & - & - & - & - & 45.00 & 22.50 & - & - & - & 11.25 & - & $\begin{array}{l}25.11 \\
\pm 0.01\end{array}$ & $\begin{array}{l}15.60 \\
\pm 0.01\end{array}$ & $\begin{array}{l}10.46 \\
\pm 0.02\end{array}$ \\
\hline 2 & - & $\begin{array}{l}16.97 \pm \\
1.85\end{array}$ & - & - & $\begin{array}{l}7.72 \pm \\
1.53\end{array}$ & - & - & - & - & $\begin{array}{l}6.96 \pm \\
0.08\end{array}$ & - & 22.50 & - & - & 22.50 & - & - & - & - & 11.25 & $\begin{array}{l}25.01 \\
\pm 0.02\end{array}$ & $\begin{array}{l}14.68 \\
\pm 0.02\end{array}$ & $\begin{array}{l}10.19 \\
\pm 0.02\end{array}$ \\
\hline 3 & - & $\begin{array}{l}15.58 \pm \\
3.76\end{array}$ & - & $\begin{array}{l}15.96 \pm \\
0.03\end{array}$ & $\begin{array}{l}28.26 \pm \\
5.52\end{array}$ & $\begin{array}{l}9.76 \pm \\
1.94\end{array}$ & $\begin{array}{l}11.13 \pm \\
1.35\end{array}$ & - & $\begin{array}{l}9.67 \pm \\
0.86\end{array}$ & - & - & 11.25 & - & 5.63 & 11.25 & 11.25 & 22.50 & - & 22.50 & - & $\begin{array}{l}36.34 \\
\pm 0.02\end{array}$ & $\begin{array}{l}17.34 \\
\pm 0.01\end{array}$ & $\begin{array}{l}13.05 \\
\pm 0.02\end{array}$ \\
\hline 4 & - & - & - & - & $\begin{array}{l}12.32 \pm \\
0.15\end{array}$ & $\begin{array}{l}9.55 \pm \\
0.71\end{array}$ & $\begin{array}{l}11.18 \pm \\
0.28\end{array}$ & - & $\begin{array}{l}9.58 \pm \\
0.24\end{array}$ & $\begin{array}{l}8.84 \pm \\
0.02\end{array}$ & - & - & - & - & 45.00 & 45.00 & 45.00 & - & 22.50 & 45.00 & $\begin{array}{l}30.67 \\
\pm 0.02\end{array}$ & $\begin{array}{l}19.50 \\
\pm 0.01\end{array}$ & $\begin{array}{l}19.38 \\
\pm 0.02\end{array}$ \\
\hline 5 & - & - & - & - & - & - & - & - & $\begin{array}{l}9.89 \pm \\
0.65\end{array}$ & - & - & - & - & - & - & - & - & - & 45.00 & - & $\begin{array}{l}30.02 \\
\pm 0.02\end{array}$ & $\begin{array}{l}18.61 \\
\pm 0.02\end{array}$ & $\begin{array}{l}14.07 \\
\pm 0.01\end{array}$ \\
\hline 6 & $\begin{array}{l}10.16 \pm \\
1.17\end{array}$ & $\begin{array}{l}16.42 \pm \\
0.12\end{array}$ & - & - & $\begin{array}{l}30.24 \pm \\
0.01\end{array}$ & $\begin{array}{l}8.68 \pm \\
2.18\end{array}$ & $\begin{array}{l}10.47 \pm \\
0.92\end{array}$ & - & - & $\begin{array}{l}7.48 \pm \\
0.03\end{array}$ & 22.50 & 90.00 & - & - & 45.00 & 90.00 & 45.00 & - & - & 11.25 & $\begin{array}{l}36.86 \\
\pm 0.01\end{array}$ & $\begin{array}{l}19.35 \\
\pm 0.01\end{array}$ & $\begin{array}{l}13.87 \\
\pm 0.02\end{array}$ \\
\hline 7 & $\begin{array}{l}11.70 \pm \\
0.63\end{array}$ & $\begin{array}{l}13.56 \pm \\
2.77\end{array}$ & - & $\begin{array}{l}9.15 \pm \\
0.01\end{array}$ & $\begin{array}{l}13.96 \pm \\
0.07\end{array}$ & $\begin{array}{l}9.07 \pm \\
0.59\end{array}$ & $\begin{array}{l}10.73 \pm \\
1.40\end{array}$ & - & $\begin{array}{l}9.59 \pm \\
0.06\end{array}$ & $\begin{array}{l}6.76 \pm \\
0.09\end{array}$ & 11.25 & 5.63 & - & 45.00 & 11.25 & 22.50 & 22.50 & - & 45.00 & 11.25 & - & $\begin{array}{l}18.88 \\
\pm 0.01\end{array}$ & $\begin{array}{l}16.31 \\
\pm 0.02\end{array}$ \\
\hline 8 & $\begin{array}{l}9.96 \pm \\
0.28\end{array}$ & - & - & - & $\begin{array}{l}29.32 \pm \\
1.19\end{array}$ & $\begin{array}{l}9.25 \pm \\
0.35\end{array}$ & - & - & $\begin{array}{l}11.06 \pm \\
0.13\end{array}$ & $\begin{array}{l}13.17 \pm \\
1.45\end{array}$ & 5.63 & - & - & - & 5.63 & 11.25 & - & - & 11.25 & 22.50 & $\begin{array}{l}34.50 \\
\pm 0.02\end{array}$ & $\begin{array}{l}13.28 \\
\pm 0.02\end{array}$ & $\begin{array}{l}10.93 \\
\pm 0.01\end{array}$ \\
\hline 9 & - & $\begin{array}{l}10.08 \pm \\
2.21\end{array}$ & - & $\begin{array}{l}7.63 \pm \\
0.64\end{array}$ & $\begin{array}{l}18.26 \pm \\
2.12\end{array}$ & - & - & - & $\begin{array}{l}11.16 \pm \\
0.14\end{array}$ & $\begin{array}{l}6.96 \pm \\
0.08\end{array}$ & - & 11.25 & - & 22.50 & 45.00 & - & - & - & 11.25 & 5.63 & $\begin{array}{l}34.05 \\
\pm 0.02\end{array}$ & $\begin{array}{l}13.99 \\
\pm 0.02\end{array}$ & $\begin{array}{l}11.51 \\
\pm 0.01\end{array}$ \\
\hline 10 & - & $\begin{array}{l}15.65 \pm \\
2.08\end{array}$ & - & $\begin{array}{l}9.59 \pm \\
1.68\end{array}$ & $\begin{array}{l}11.76 \pm \\
3.22\end{array}$ & $\begin{array}{l}10.92 \pm \\
1.44\end{array}$ & $\begin{array}{l}12.98 \pm \\
1.99\end{array}$ & - & $\begin{array}{l}10.68 \pm \\
0.54\end{array}$ & $\begin{array}{l}8.45 \pm \\
0.09\end{array}$ & - & 22.50 & - & 22.50 & 5.63 & 11.25 & 45.00 & - & 22.50 & 22.50 & $\begin{array}{l}12.32 \\
\pm 0.02\end{array}$ & $\begin{array}{l}23.04 \\
\pm 0.02\end{array}$ & $\begin{array}{l}19.92 \\
\pm 0.01\end{array}$ \\
\hline 11 & $\begin{array}{l}9.06 \pm \\
0.88\end{array}$ & - & - & $\begin{array}{l}10.98 \pm \\
0.13\end{array}$ & $\begin{array}{l}12.12 \pm \\
0.51\end{array}$ & - & - & - & $\begin{array}{l}10.43 \pm \\
0.36\end{array}$ & $\begin{array}{l}8.23 \pm \\
0.11\end{array}$ & 22.50 & - & - & 22.50 & 11.25 & - & - & - & 22.50 & 22.50 & - & - & - \\
\hline 12 & $\begin{array}{l}20.37 \pm \\
0.75\end{array}$ & $\begin{array}{l}22.50 \pm \\
2.59\end{array}$ & - & - & - & $\begin{array}{l}11.48 \pm \\
1.95\end{array}$ & $\begin{array}{l}16.71 \pm \\
1.01\end{array}$ & - & $\begin{array}{l}11.13 \pm \\
0.43\end{array}$ & $\begin{array}{l}6.36 \pm \\
0.02\end{array}$ & 11.25 & 11.25 & - & - & - & 22.50 & 22.50 & - & 22.50 & 11.25 & - & - & - \\
\hline 13 & - & - & - & - & - & $\begin{array}{l}10.08 \pm \\
0.43\end{array}$ & $\begin{array}{l}9.87 \pm \\
1.34\end{array}$ & - & $\begin{array}{l}9.87 \pm \\
0.38\end{array}$ & $\begin{array}{l}8.91 \pm \\
0.05\end{array}$ & - & - & - & - & - & 11.25 & 22.50 & - & 22.50 & 11.25 & $\begin{array}{l}25.41 \\
\pm 0.02\end{array}$ & $\begin{array}{l}13.87 \\
\pm 0.01\end{array}$ & $\begin{array}{l}10.51 \\
\pm 0.02 \\
\end{array}$ \\
\hline
\end{tabular}

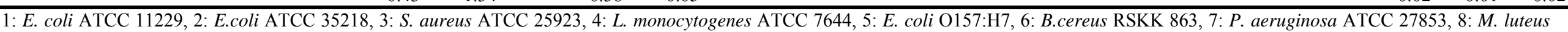
NRRL B-4375, 9: S. sonnei Mu:57, 10: Y. enterocolitica NCTC 11175, 11: C. albicans ATCC 10231, 12: S.cerevisiae (grape isolate), 13: S. enteritidis ATCC 13076

-: no zone of inhibition

${ }^{a}$ diameter of the inhibition zone including disc diameter. Values are means \pm SD of three seperate experiments done in triplicate. 
http://dx.doi.org/10.4314/ajtcam.v10i3.11

Table 2: Scavenging activity (\%) on DPPH radicals of different Allium scabriflorum and Allium tchihatschewii extracts and standart antioxidants at different concentrations

\begin{tabular}{|c|c|c|c|c|c|c|c|c|c|c|c|c|c|c|}
\hline \multirow{3}{*}{ Concentration } & \multicolumn{14}{|c|}{ DPPH Scavenging Activity $(\%)$} \\
\hline & \multicolumn{4}{|c|}{ Allium scabriflorum } & \multicolumn{4}{|c|}{ Allium tchihatschewii } & \multicolumn{3}{|c|}{ Standards } & \multirow[b]{2}{*}{ BHA } & \multirow[b]{2}{*}{ BHT } & \multirow[b]{2}{*}{$\begin{array}{l}\text { Vitamin } \\
\text { C }\end{array}$} \\
\hline & ME & $\mathrm{EE}$ & WE & $\mathrm{HE}$ & DCM-E & ME & $\mathrm{EE}$ & WE & $\mathrm{HE}$ & DCM-E & $\alpha$-Tocopherol & & & \\
\hline $5 \mu \mathrm{g} / \mathrm{mL}$ & $\begin{array}{l}18.15 \\
\pm 0.15\end{array}$ & $\begin{array}{l}17.44 \\
\pm 0.09\end{array}$ & $\begin{array}{l}12.10 \\
\pm 0.16\end{array}$ & $\begin{array}{l}14.59 \\
\pm 0.06\end{array}$ & $\begin{array}{l}13.88 \\
\pm 0.05\end{array}$ & $\begin{array}{l}11.74 \\
\pm 0.16\end{array}$ & $\begin{array}{l}12.81 \\
\pm 0.02\end{array}$ & $\begin{array}{l}12.81 \\
\pm 0.08\end{array}$ & $\begin{array}{l}12.46 \\
\pm 0.09\end{array}$ & $\begin{array}{l}1.07 \\
\pm 0.12\end{array}$ & $\begin{array}{l}89.01 \\
\pm 0.04\end{array}$ & $\begin{array}{l}86.01 \\
\pm 0.06\end{array}$ & $\begin{array}{l}56.04 \\
\pm 0.05\end{array}$ & $\begin{array}{l}92.31 \\
\pm 0.06\end{array}$ \\
\hline $25 \mu \mathrm{g} / \mathrm{mL}$ & $\begin{array}{l}23.49 \\
\pm 0.03\end{array}$ & $\begin{array}{l}27.40 \\
\pm 0.24\end{array}$ & $\begin{array}{l}12.81 \\
\pm 0.12\end{array}$ & $\begin{array}{l}17.08 \\
\pm 0.02\end{array}$ & $\begin{array}{l}22.06 \\
\pm 0.09\end{array}$ & $\begin{array}{l}27.76 \\
\pm 0.06\end{array}$ & $\begin{array}{l}14.60 \\
\pm 0.03\end{array}$ & $\begin{array}{l}14.60 \\
\pm 0.16\end{array}$ & $\begin{array}{l}16.73 \\
\pm 0.08\end{array}$ & $\begin{array}{l}1.78 \\
\pm 0.17\end{array}$ & $\begin{array}{l}94.14 \\
\pm 0.05\end{array}$ & $\begin{array}{l}94.14 \\
\pm 0.07\end{array}$ & $\begin{array}{l}93.41 \\
\pm 0.03\end{array}$ & $\begin{array}{l}94.51 \\
\pm 0.08\end{array}$ \\
\hline $50 \mu \mathrm{g} / \mathrm{mL}$ & $\begin{array}{l}28.11 \\
\pm 0.06\end{array}$ & $\begin{array}{l}37.01 \\
\pm 0.11\end{array}$ & $\begin{array}{l}15.30 \\
\pm 0.15\end{array}$ & $\begin{array}{l}20.29 \\
\pm 0.04\end{array}$ & $\begin{array}{l}57.65 \\
\pm 0.13\end{array}$ & $\begin{array}{l}39.50 \\
\pm 0.07\end{array}$ & $\begin{array}{l}15.30 \\
\pm 0.14\end{array}$ & $\begin{array}{l}18.51 \\
\pm 0.07\end{array}$ & $\begin{array}{l}19.57 \\
\pm 0.11\end{array}$ & $\begin{array}{l}2.49 \\
\pm 0.03\end{array}$ & $\begin{array}{l}95.60 \\
\pm 0.08\end{array}$ & $\begin{array}{l}95.60 \\
\pm 0.12\end{array}$ & $\begin{array}{l}97.44 \\
\pm 0.06\end{array}$ & $\begin{array}{l}96.34 \\
\pm 0.07\end{array}$ \\
\hline $75 \mu \mathrm{g} / \mathrm{mL}$ & $\begin{array}{r}32.38 \\
\pm 0.20\end{array}$ & $\begin{array}{l}43.06 \\
\pm 0.16\end{array}$ & $\begin{array}{l}17.08 \\
\pm 0.09\end{array}$ & $\begin{array}{l}22.42 \\
\pm 0.08\end{array}$ & $\begin{array}{l}61.57 \\
\pm 0.12\end{array}$ & $\begin{array}{r}46.26 \\
\pm 0.11 \\
\end{array}$ & $\begin{array}{l}16.01 \\
\pm 0.12\end{array}$ & $\begin{array}{r}19.22 \\
\pm 0.09\end{array}$ & $\begin{array}{l}28.47 \\
\pm 0.03 \\
\end{array}$ & $\begin{array}{l}3.20 \\
\pm 0.08\end{array}$ & $\begin{array}{l}97.07 \\
\pm 0.04\end{array}$ & $\begin{array}{l}97.07 \\
\pm 0.11\end{array}$ & $\begin{array}{l}99.64 \\
\pm 0.03\end{array}$ & $\begin{array}{l}99.63 \\
\pm 0.04\end{array}$ \\
\hline
\end{tabular}

a each value represents the mean of triplicate studies. 
Asan Ozusaglam et al., Afr J Tradit Complement Altern Med. (2013) 10(3):449-457

http://dx.doi.org/10.4314/ajtcam.v10i3.11

Table 3: Percentage of metal chelating and hydrogen peroxide scavenging activities of different Allium scabriflorum and Allium tchihatschewii extracts and standart antioxidants

\begin{tabular}{|c|c|c|c|c|c|c|c|c|c|c|c|c|c|c|c|c|}
\hline \multirow[b]{2}{*}{$\%$ Activity $^{\mathrm{a}}$} & \multicolumn{5}{|c|}{ Allium scabriflorum } & \multicolumn{5}{|c|}{ Allium tchihatschewii } & \multicolumn{6}{|l|}{ Standards } \\
\hline & $\mathrm{ME}$ & $\mathrm{EE}$ & WE & $\mathrm{HE}$ & DCM-E & $\mathrm{ME}$ & $\mathrm{EE}$ & WE & $\mathrm{HE}$ & DCM-E & $\alpha$-Tocopherol & BHA & BHT & $\begin{array}{l}\text { Vitamin } \\
\mathrm{C}\end{array}$ & Trolox & EDTA \\
\hline $\begin{array}{l}\text { Metal chelating } \\
(15 \mu \mathrm{g} / \mathrm{mL})\end{array}$ & $\begin{array}{l}98.96 \\
\pm 0.23\end{array}$ & $\begin{array}{l}99.19 \\
\pm 0.13\end{array}$ & $\begin{array}{l}98.92 \\
\pm 0.06\end{array}$ & $\begin{array}{l}99.42 \\
\pm 0.25\end{array}$ & $\begin{array}{l}99.69 \\
\pm 0.11\end{array}$ & $\begin{array}{l}97.64 \\
\pm 0.09\end{array}$ & $\begin{array}{l}99.73 \\
\pm 0.08\end{array}$ & $\begin{array}{l}99.00 \\
\pm 0.14\end{array}$ & $\begin{array}{l}99.42 \\
\pm 0.17\end{array}$ & $\begin{array}{l}99.69 \\
\pm 0.08\end{array}$ & $\begin{array}{l}8.33 \\
\pm 0.10\end{array}$ & $\begin{array}{l}40.91 \\
\pm 0.07\end{array}$ & $\begin{array}{l}30.30 \\
\pm 0.04\end{array}$ & $\begin{array}{l}60.61 \\
\pm 0.08\end{array}$ & $\begin{array}{l}11.36 \\
\pm 0.02\end{array}$ & $\begin{array}{l}84.09 \\
\pm 0.09\end{array}$ \\
\hline $\begin{array}{l}\mathrm{H}_{2} \mathrm{O}_{2} \\
\text { scavenging }(30 \\
\mu \mathrm{g} / \mathrm{mL})\end{array}$ & $\begin{array}{l}82.61 \\
\pm 0.27\end{array}$ & $\begin{array}{l}77.36 \\
\pm 0.09\end{array}$ & $\begin{array}{l}83.58 \\
\pm 0.43\end{array}$ & $\begin{array}{l}80.53 \\
\pm 0.12\end{array}$ & $\begin{array}{l}67.43 \\
\pm 0.14\end{array}$ & $\begin{array}{l}82.61 \\
\pm 0.04\end{array}$ & $\begin{array}{l}84.66 \\
\pm 0.09\end{array}$ & $\begin{array}{l}84.81 \\
\pm 0.25\end{array}$ & $\begin{array}{l}79.44 \\
\pm 0.24\end{array}$ & $\begin{array}{l}82.26 \\
\pm 0.12\end{array}$ & $\begin{array}{l}42.85 \\
\pm 0.08\end{array}$ & $\begin{array}{l}0.73 \\
\pm 0.11\end{array}$ & $\begin{array}{l}37.60 \\
0 \pm 0.05\end{array}$ & $\begin{array}{l}86.17 \\
\pm 0.03\end{array}$ & $\begin{array}{l}1.55 \\
\pm 0.03\end{array}$ & $\mathrm{Ns}^{\mathrm{b}}$ \\
\hline
\end{tabular}

${ }^{a}$ each value represents the mean of triplicate studies; ${ }^{b}$ Not studied

Table 4: Total phenolic and flavonoid contents of different Allium scabriflorum and Allium tchihatschewii extracts

\begin{tabular}{|c|c|c|c|c|c|c|c|c|c|c|}
\hline \multirow[b]{2}{*}{ Antioxidant Components } & \multicolumn{5}{|c|}{ Allium scabriflorum } & \multicolumn{5}{|c|}{ Allium tchihatschewii } \\
\hline & $\mathrm{ME}$ & $\mathrm{EE}$ & WE & $\mathrm{HE}$ & DCM-E & $\mathrm{ME}$ & EE & WE & $\mathrm{HE}$ & DCM-E \\
\hline \multirow[t]{2}{*}{ Phenolics $^{\mathrm{a}}(\mu \mathrm{gGAE} / \mathrm{mg}$ exract) } & 28.25 & 30.20 & 28.25 & $27.42 \pm$ & $33.25 \pm$ & $29.36 \pm$ & $27.69 \pm$ & $29.64 \pm$ & $46.86 \pm$ & $27.42 \pm$ \\
\hline & \pm 0.01 & \pm 0.02 & \pm 0.01 & 0.09 & 0.03 & 0.08 & 0.01 & 0.01 & 0.02 & 0.11 \\
\hline \multirow[t]{2}{*}{ Flavonoids $^{\mathrm{a}}(\mu \mathrm{gQE} / \mathrm{mg}$ exract) } & $1.42 \pm$ & $11.23 \pm$ & $1.79 \pm$ & $8.02 \pm$ & $44.06 \pm$ & $9.15 \pm$ & $0.85 \pm$ & $0.66 \pm$ & $17.83 \pm$ & $12.74 \pm$ \\
\hline & 0.01 & 0.06 & 0.01 & 0 & 0.03 & 0.02 & 0 & 0 & 0.14 & 0.07 \\
\hline
\end{tabular}

${ }^{a}$ each value represents the mean of triplicate studies. 
comparison with Ampicillin. Only the DCM extract of A. tchihatschewii has shown better antibacterial activity against $M$. luteus NRRL B-4375 when compared with standard Gentamicin. It is interesting to note that the extracts are not pure compounds and in spite of it, good results were obtained, which only suggests the potential of these extracts. Hence, some $A$. scabriflorum and A. tchihatschewii extracts could be used as new natural antimicrobial ingredients for the food industry.

Minimum inhibitory concentration (MIC) is a quantitative endpoint measurement which is most commonly used for evaluating the antimicrobial effect of antibiotics or extracts. MIC values for microorganisms which were sensitive to extracts of A. scabriflorum and A. tchihatschewii were determined. MIC values of A. scabriflorum and A. tchihatschewii extracts were in the range of 5.63-45.00 $\mu \mathrm{g} / \mu \mathrm{L}$ and $11.25-90.00 \mu \mathrm{g} / \mu \mathrm{L}$, respectively. The both extracts (methanol and DCM) of $A$. scabriflorum which were sensitive to M. luteus NRRL B-4375 had the lowest MIC $(5.63 \mu \mathrm{g} / \mu \mathrm{L})$ values. Two other extracts (n-hexane and DCM) of the species showed the same MIC value. The methanol extract of A. tchihatschewii, which had low inhibitory activity in the disc diffusion method, had the highest MIC $(90.00 \mu \mathrm{g} / \mu \mathrm{L})$ values. Conclusively, as can be seen from the Table 1, A. scabriflorum and A. tchihatschewii extracts showed various degrees of antimicrobial activity depending on the tested microorganisms, solvents and plant species.

Previous studies showed high antibacterial activity of sulfur and other numerous phenolic compounds found in Allium plants (Rivlin, 2001; Griffiths et al., 2002). Aroma components of Allium species are physiologically active and exhibit a widespread possibility of application. There is ample evidence that many sulfur compounds found in Allium tissue preparations are responsible for various biological effects such as antimicrobial (antibacterial, antifungal, antiviral), antiprotozoal, antioxidant, antitumour, antihypertensive, hypolipidaemic, hepatoprotective andantithrombotic activities (Rose et al., 2005; Tapiero et al., 2004).

\section{Antioxidant capacity}

In this research, three most widely used complementary test systems, namely DPPH free radical scavenging activity, metal chelating and hydrogen peroxide scavenging assays, were applied to evaluate the antioxidant capacities of $A$. scabriflorum and A. tchihatschewii. The results were given in Table 2 and 3. In the DPPH test, the extracts obtained using different solvents from A. scabriflorum and A. tchihatschewii were all able to inhibit the DPPH (at concentrations ranging from 5 to $75 \mu \mathrm{g} / \mathrm{mL}$ ) (Table 2). The antioxidant potential varied widely and ranged from $17.08-61.57 \%$ and from 3.20 $46.26 \%$ at $75 \mu \mathrm{g} / \mathrm{mL}$ concentration of A. scabriflorum and A. tchihatschewii, respectively. The radical scavenging of BHT and BHA, synthetic antioxidants used in food industry, was higher than the extracts of A. scabriflorum and A. tchihatschewii, $97.07 \%$ and $99.64 \%$, respectively. When compared to all extracts, the natural antioxidants such as ascorbic acid and $\alpha-$ tocopherol showed better radical scavenging activities as with synthetic antioxidants. Free radicals cause auto oxidation of unsaturated lipids in food (Kaur and Perkins, 1991), and the radical scavenging system of the extracts could be attributed to their hydrogen donating ability. Tepe et al. (2005) found that, of the methanol extracts of five Allium species, namely A. nevsehirense Koyuncu et Kollmann, A. sivasicum N. Özhatay et Kollmann, A. dictyoprasum Ledep, A. scorodoprasum L. subsp. rotundum (L.) Stearn and A. atroviolaceum Boiss.; the first two are endemic for the Turkish flora, showed a strong radical scavenging activity against DPPH when compared to synthetic antioxidant BHT. The radical scavenging values, however, depended on plant species, locality and polarity of extraction solvents.

The ferrous ion chelating activities of $\alpha$-tocopherol, BHA, BHT, Vitamin C, trolox, EDTA and extracts of $A$. scabriflorum and A. tchihatschewii are shown in Table 3. It has been well recognized that transition metal ions such as those of iron and copper are important catalysts for the generation of the first few free radicals to initiate the radical chain reaction or the radical mediates lipid peroxidation (Nawar, 1996). Chelating agents may inhibit radical generations by stabilizing transition metals, consequently reducing free radical damage. The chelating activity of the plants was evaluated against $\mathrm{Fe}^{2+}$ to estimate the potential antioxidant activities of the two plant extracts and standards. The metal scavenging capacities of 15 $\mu \mathrm{g} / \mathrm{mL}$ doses of all tested extracts of A. scabriflorum and A. tchihatschewii were found to be marked higher when comparing to all standards at the same concentration. Table 3 reveals that all the extracts of A. scabriflorum and A. tchihatschewii demonstrate a marked capacity for iron binding, suggesting their action as lipid peroxidation protector.

The scavenging abilities of various extracts of A. scabriflorum and A. tchihatschewii on hydrogen peroxide is shown in Table 3 and compared with that of $\alpha$-tocopherol, BHA, BHT, ascorbic acid and trolox as standards. The extracts (30 $\mu \mathrm{g} / \mathrm{mL}$ ) of A. scabriflorum exhibited 83.58 (water), 82.61 (methanol), 80.53 (n-hexane), 77.36 (ethanol) and 67.43 (DCM) \% scavenging activities on hydrogen peroxide. On the other hand, A. tchihatschewii extracts showed 84.81 (water), 84.66 (ethanol), 82.61 (methanol), 82.26 (DCM) and 79.44 (n-hexane) \% hydrogen peroxide scavenging activities at the same dose. The results show that both $A$. scabriflorum and A. tchihatschewii extracts have stronger hydrogen peroxide scavenging activities. Those values are higher than those of $\alpha$-tocopherol, BHA, BHT and trolox, but close to that of ascorbic acid. Hydrogen peroxide itself is not very reactive, but it is sometimes is toxic to cells because it may give rise to hydroxyl radical in the cells (Halliwell, 1991). Therefore, removal of $\mathrm{H}_{2} \mathrm{O}_{2}$ is very important for antioxidant defence in cell or food systems. Dietary polyphenols have also been shown to protect mammalian and bacterial cells from cytotoxicity induced by hydrogen peroxide, especially compounds with the orthodihydroxy phenolic structures such as quercetin, catechin, gallic acid ester, caffeic acid ester (Nakayama, 1994; Nakayama et al., 1993).

\section{Total phenolics and flavonoids}

Total phenolics ( $\mu \mathrm{g}$ GAE/mg extracts) and flavanoids ( $\mu \mathrm{g} \mathrm{QE} / \mathrm{mg}$ extracts) of A. scabriflorum and A. tchihatschewii extracts were shown in Table 4.

As shown in Table 4, there are large variations in the total phenolic content of the plant species investigated, ranging from 27.42 to 33.25 and from 27.69 to $46.86 \mu \mathrm{g}$ GAE/mg dry extracts for those of A. scabriflorum and A. tchihatschewii extracts, respectively. Mladevonić et al. (2011) reported that the total phenolic was found as $45.39 \mathrm{mg}$ GAE/g 
in A. porrum L. dry extract. According to our findings, total phenolics of A. scabriflorum and A. tchihatschewii extracts, except $A$. tchihatschewii n-hexane extract ( $46.86 \mu \mathrm{g} \mathrm{GAE} / \mathrm{mg}$ extract), were lower than that of the A. porrum L. leaf extract.

The key role of phenolic compounds as scavengers of free radicals is emphasized in several reports (Komali et al., 1999; Moller et al., 1999). Phenols are important components of plants. They were reported to eliminate radicals due to their hydroxyl groups (Hatano et al., 1989), and they contribute directly to antioxidant effect of system (Duh et al., 1999). Polyphenolic compounds have an important role in stabilizing lipid oxidation and are associated with antioxidant activity (Cakir et al., 2003; Wagner et al., 1992). The phenolic compounds may contribute directly to antioxidative action (Duh et al., 1999).

It has been reported that compounds such as the flavonoids, which contain hydroxyls, are responsible for the radical scavenging effects of most plants (Das and Pereira, 1990). The mechanisms of action of the flavonoids are through scavenging or chelating process (Cook and Samman, 1996). According to our findings, flavonoid contents of A. scabriflorum extracts are higher than those of A. tchihatschewii. The total flavonoid contents of A. scabriflorum and A. tchihatschewii varied from 1.42 to 44.06 and from 0.66 to $17.83 \mu \mathrm{gQE} / \mathrm{mg}$, respectively (Table 4).

\section{Conclusion}

Antioxidant and antimicrobial properties of extracts from many plants are of great interest in the food industry, since their potential use as natural additives can fulfill a growing market for replacements of synthetic substances with natural sources. Therefore, new plant species, as natural sources, could be introduced for this purpose. From this point of view, our study may be considered as a new report based on antimicrobial and antioxidative potentials of $A$. scabriflorum and $A$. tchihatschewii growing wild in the Turkish flora and they could be evaluated as a starting point for further investigations.

\section{Acknowledgments}

The authors are grateful to the Scientific and Technological Research Council of Turkey (TUBITAK). This research was supported by a grant from, TUBITAK, TBAG (Research Grant No. 110T495).

\section{References}

1. Blois, M.S. (1958). Antioxidant determinations by the use of a stable free radical. Nature 181: 1199-1200.

2. Cakir, A., Mavi, A., Yıldırım, A., Duru, M. E., Harmandar, M. and Kazaz, C. (2003). Isolation and characterization of antioxidant phenolic compounds from the aerial parts of Hypericum hyssopifolium L. by activity-guided Fractionation. J. Ethnopharm. 87: 7383.

3. Cao, G., Soc, E. and Prior, R. L. (1996). Antioxidant capacity of tea and common vegetables. J. Agr. Food. Chem. 44: 3426-3431.

4. Cook, N. C. and Samman, S. (1996). Flavonoids-chemistry, metabolism, cardioprotective effects, and dietary sources. J. Nutr. Biochem. 7: 66-76.

5. Das, N. P. and Pereira, T. A. (1990). Effect of flavonoids on thermal autoxidation of palm oil: structural activity relationships. J. Am. Oil. Chem. Soc. 67: 255-258.

6. Davis, P. H., Tan, K. and Mill, R.R. (1988). Flora of Turkey and the East Aegean Islands, Vol. 10. Edinburgh University Press, Edinburgh pp. 221-223.

7. Deak, T. and Beuchat, L. R. (1996). Handbook of food spoilage. CRC Press, New York, USA

8. Dinis, T. C. P., Madeira, V. M. C. and Almeida, L. M. (1994). Action of phenolic derivatives (Acetaminophen, Salicylate, and 5aminosalicylate) assay nhibitors of membrane lipid peroxidation and assay peroxyl radical scavengers. Arch. Biochem. Biophys. 315: 161-169.

9. Duh, P. D., Tu, Y. Y. and Yen, G. C. (1999). Antioxidant activity of water extract of harn jyur Chyrsanthemum morifolium (Ramat). Lebensm. Wiss. Technol. 32: 269-277.

10. Durmaz, H., Sagun, E., Tarakci, Z. and Ozgokce, F. (2006). Antibacterial activities of Allium vineale, Chaerophyllum macropodum and Prangos ferulacea. Afr. J. Biotechnol. 5: 1795-1798.

11. Friesen, N., Fritsch, R. M. and Blattner, F. R. (2006). Phylogeny and new intrageneric classification of Allium L. (Alliaceae) based on nuclear ribosomal DNA ITS sequences. Aliso 22: 372-395.

12. Fritsch, R. M. and Friesen, N. (2002). Evolution, domestication and taxonomy. In: Rabinowitch, D. and Currah, L. (eds) Allium Crop Science: Recent Advances. CAB International Wallingford, UK pp. 5-30.

13. Gao, J. J., Igalashi, K. and Nukina, M. (1999). Radical scavenging activity of phenylpropanoid glycosides in Caryop- teris incana. Biosci. Biotech. Biochem. 63: 983-988.

14. Gazzani, G., Papetti, A., Daglia, M., Berte, F. and Gregotti, C. (1998). Protective activity of water soluble components of some common diet vegetables on rat liver microsome and the effect of thermal treatment. J. Agr. Food. Chem. 46: 4123-4127.

15. Griffiths, G., Trueman, L., Crowther, T., Thomas, B. and Smith, B. (2002). Onionsa global benefits to health. Phytother. Res. 16: 603-615.

16. Halliwell, B. (1991). Reactive oxygen species in living systems: source, biochemistry, and role in human disease. Am. J. Med. 91: $14-22$.

17. Hatano, T., Edamatsu, R., Mori, A., Fujita, Y. and Yasuhara, E. (1989). Effect of interaction of tannins with co-existing substances. VI. Effects of tannins and related polyphenols on superoxide anion radical and on DPPH radical. Chem. Pharm. Bull. 37: 2016-2021.

18. Kaur, H. and Perkins, J. (1991). The free radical chemistry of food additives. In: Aruoma, O.I. and Halliwell, B. (eds) Free radicals and food additives. Taylor and Francis Ltd., London pp. 17-35.

19. Kollmann, N. F. (1984). Allium L. In: Davis, P. H. (ed) Flora of Turkey and the East Aegean Islands. Edinburgh University Press, Edinburgh pp. 98-211. 
20. Komali, A. S., Zheng, Z. and Shetty, K. (1999). A mathematical model for the growth kinetics and synthesis of phenolics in oregano (Origanum vulgare) shoot cultures inoculated with Pseudomonas species. Process. Biochem. 35: $227-235$.

21. Koneman, E. W., Allen, S. D., Janda, W. M., Scherckenberger, P. C. and Winn, W. C. (1997). Color Atlas and Textbook of Diagnostic Microbiology. Lippincott-Raven, Philadelphia.

22. Martin, G. J. (1995). Ethnobotany: A Methods Manual. Chapman and Hall, London.

23. Mladenović, J. D., Mašković, P. Z., Pavlović, R. M., Radovanović, B. C. , Aćamović-Đoković, G. and Cvijović, M. S. (2011). Antioxidant activity of ultrasonic extracts of leek Allium porrum L. Hem. Ind. 65: 473-477.

24. Moller, J. K. S., Madsen, H. L., Altonen, T. and Skibsted, L.H. (1999). Dittany (Origanum dictamnus) as a source of waterextractable antioxidants. Food Chem., 64: 215-219.

25. Murray, P. R., Baron, E.J., Pfaller, M. A., Tenover, F.C. and Yolke, R.H. (1995). Manual of Clinical Microbiology, 6th edn. ASM, Washington, DC.

26. Najjaa, H., Fattouch, S., Ammar, E. and Neffati, M. (2011). Antimicrobial potentials of Allium roseum: Recent Advances and Trends. In: Mendez-Vilas, A. (ed) Science against microbial pathogens: communicating current research and technological advances. Formatex pp. 1205-1210.

27. Najjaa, H., Neffati, M., Zouari, S. and Emmar, E. (2007). Essential oil composition and antibacterial activity of different extract of Allium roseum L a North African endemic species. C. R. Chim. 10: 820-826.

28. Nakayama, T. (1994). Suppression of hydroperoxide-induced cytotoxicity by polyphenols. Cancer. Res. 54: 1991s-1993s.

29. Nakayama, T., Yamaden, M., Osawa, T. and Kawakishi, S. (1993). Suppression of active oxygen-induced cytotoxicity by flavonoids. Biochem. Pharmacol. 45: 265-267.

30. Nawar, W. W. (1996). Lipids. In: Fennema, O. R. (ed) Food chemistry. Marcel Dekker, New York pp. $225-313$.

31. Osawa, T. and Namiki, M. (1981). A novel type of antioxidant isolated from leaf wax of Eucalyptus Leaves. Agric. Biol. Chem. 45: 735-739.

32. Özhatay, F. N., Kültür, Ş. and Gürdal M. B. (2011). Chect-List of additional taxa to the supplement Flora of Turkey V. Turk. J. Bot. 35: 589-624.

33. Özhatay, N., Kültür, Ş. and Aslan, S. (2009). Chect-List of additional taxa to the supplement Flora of Turkey IV. Turk. J. Bot. 33: 191-226.

34. Özhatay, N and Kültür, Ş (2006). Chect-List of additional taxa to the supplement Flora of Turkey III. Turk. J. Bot. 30: 281-316.

35. Özhatay, N and Tzanoudakis, D (2000). Allium L. In: Güner, A., Özhatay, N., Ekim, T. and Başer, K. (eds) Flora of Turkey and the East Aegean Islands, (vol 11). Edinburgh University Press, Edinburgh pp. 224-232.

36. Parekh, J and Chanda, S (2007). In vitro antimicrobial activity of Trapa natans L. fruit rind extracted in different solvents. Afr. J. Biotechnol. 6:766-770.

37. Park, Y. K., Koo, M. H., Ikegaki, M. and Contado, J. L. (1997). Comparison of the flavonoid aglycone contents of Apis mellifera propolis from various regions of Brazil. Arq. Biol. Tecnol. 40: 97-106.

38. Paz, E. A., Cerdeiras, M. P., Fernandez, J., Ferreira, F., Moyna, P., Soubes, M., Vázquez, A., Vero, S. and Zunino, L. (1995). Screening of Uruguayan medicinal plants for antimicrobial activity. J. Ethnopharm. 45: 67-70.

39. Rauha, J.-P., Remesa, S., Heinonenb, M., Hopiab A., Kähkönenb M., Kujalac T., Pihlajac K., Vuorelaa, H. and Vuorelaa, P. (2000). Antimicrobial effects of Finnish plant extracts containing flavonoids and other phenolic compounds. Int. J. Food. Microbiol. 56: 3-12.

40. Rose, P., Whiteman, M., Moore, P. K. and Zhu, Y. Z. (2005). Bioactive S-alk(en)yl cysteine sulfoxide metabolites in the genus Allium: the chemistry of potential therapeutic agents. Nat. Prod. Rep. 22: 351-368.

41. Rivlin, R. S. (2001). Historical perspective on the use of garlic. J. Nutr. 131: 951-954.

42. Ruch, R. J., Cheng, S. J. and Klaunig, J. E. (1989). Prevention of cytotoxicity and inhibition of intracellular comunication by antioxidant catechins isolated from Chinese grean tea. Carcinogenesis 10: 1003-1008.

43. Sağdıç, O. and Özcan, M. (2003). Antibacterial activity of Turkish spice hydrosols. Food Control 14: $141-143$.

44. Singleton, V. L. and Rossi, J. A. (1965). Colorimetry of total phenolics with phosphomolybdic-phosphotungstic acid reagents. Am. J. Enol. Viticult. 16: 144-158.

45. Şahin, F., Karaman, İ., Güllüce, M., Ögütçü, H., Şengül, M., Adıgüzel, A., Öztürk, S. and Kotan, R. (2003). Evaluation of antimicrobial activities of Satureja hortensis L. J. Ethnopharm. 87: 61-65.

46. Tapiero, H., Townsend, D. and Tew, K. (2004). Organosulfur compounds from Alliaceae in the prevention of human pathologies. Biomed. Pharmacother. 58: 183-193.

47. Tepe, B., Sokmen, M., Akpulat, H. A. and Sokmen, A (2005). In vitro ntioxidant activities of the methanol extracts of five Allium species from Turkey. Food Chem. 92: 89-92.

48. Vlientinck, A. J., van Hoof, L., Totté, J., Lasure, A., Vanden Berghe, D., Rwangabo, P. C. and Mvukiyumwami, J. (1995). Screening of hundred Rwandese medicinal plants for antimicrobial and antiviral properties. J. Ethnopharm. 46: 31-47.

49. Wagner, J. R., Hu, C. C. and Ames, B. N. (1992). Endogenous oxidative damage of deoxycytidine in DNA. Proc. Nat. Acad. Sci. USA 89: 3380-3384.

50. Williams, G. M., Iatropoulos, M. J. and Whysner, J. (1999). Safety assessment of butylated hydroxyanisole and butylated hydroxyltoluene as antioxidant food additives. Food Chem. Toxicol. 37: 1027-1038.

51. Yin, M. C. and Cheng, W. S. (1998). Antioxidant activity of several Allium members. J Agr Food Chem. 46: 4097-4101.

52. Zeng W. -C., Jia L. -R., Zhang Y., Cen J. -Q., Chen X., Gao, H., Feng, S. and Huang, Y.-N. (2011). Antibrowning and antimicrobial activities of the water-soluble extract from pine needles of Cedrus deodara. J. Food Sci. 76: C318-C323. 